Article

\title{
Quantifying Dynamical Complexity of Magnetic Storms and Solar Flares via Nonextensive Tsallis Entropy
}

\author{
Georgios Balasis $^{1, \star}$, Ioannis A. Daglis ${ }^{1}$, Constantinos Papadimitriou ${ }^{1,2}$, \\ Anastasios Anastasiadis ${ }^{1}$, Ingmar Sandberg ${ }^{1}$ and Konstantinos Eftaxias ${ }^{3}$ \\ ${ }^{1}$ Institute for Space Applications and Remote Sensing, National Observatory of Athens, Metaxa and \\ Vasileos Pavlou, Penteli 15236, Athens, Greece; E-Mails: daglis@noa.gr (I.A.D.); \\ magekoylen@yahoo.com (C.P.); anastasi@noa.gr (A.A.); sandberg@noa.gr (I.S.) \\ ${ }^{2}$ Section of Astrophysics, Astronomy and Mechanics, Department of Physics, University of Athens, \\ Panepistimiopolis, Zografos 15784, Athens, Greece \\ ${ }^{3}$ Section of Solid State Physics, Department of Physics, University of Athens, Panepistimiopolis, \\ Zografos 15784, Athens, Greece; E-Mail: ceftax@phys.uoa.gr \\ * Author to whom correspondence should be addressed; E-Mail: gbalasis@ @oa.gr; \\ Tel.: +30-210-8109114; Fax: +30-210-6138343.
}

Received: 1 September 2011; in revised form: 28 September 2011 / Accepted: 30 September 2011 / Published: 14 October 2011

\begin{abstract}
Over the last couple of decades nonextensive Tsallis entropy has shown remarkable applicability to describe nonequilibrium physical systems with large variability and multifractal structure. Herein, we review recent results from the application of Tsallis statistical mechanics to the detection of dynamical changes related with the occurrence of magnetic storms. We extend our review to describe attempts to approach the dynamics of magnetic storms and solar flares by means of universality through Tsallis statistics. We also include a discussion of possible implications on space weather forecasting efforts arising from the verification of Tsallis entropy in the complex system of the magnetosphere.
\end{abstract}

Keywords: Tsallis entropy; magnetospheric dynamics; magnetic storms; solar flares; space weather 


\section{Introduction}

Nonextensive statistical mechanics through Tsallis entropy provides a solid theoretical basis for describing and analyzing complex systems out of equilibrium, systems exhibiting long-range correlations, memory, or fractal properties [1-3]. Nowadays, mounting evidence of experimental, observational and computational verifications and applications of the Nonextensive Statistical Mechanics and Thermodynamics [4] as well as theoretical verifications and applications of the theory [5] exist. The reader is referred to [6] for a complete list of publications ( $\sim 3500)$ related to the topic. Among the works listed there are papers on the heliosphere [7,8], solar wind [9,10] and magnetospheric dynamics [11-14].

For instance, Leubner and Vörös $[9,10]$ approached solar wind turbulence and intermittency based on nonextensive Tsallis entropy concepts. The nonextensive approach assured for experimental studies of solar wind intermittency independence from influence of a priori model assumptions $[9,10]$. It was argued that the intermittency of the turbulent fluctuations should be related physically to the nonextensive character of the interplanetary medium counting for nonlocal interactions via the entropy generalization [9,10]. Moreover, the nonextensive Tsallis entropy has been recently introduced [11-14] as an appropriate information measure to investigate dynamical complexity in the magnetosphere. The method has been employed for analyzing $D_{s t}$ time series and gave promising results, detecting the complexity dissimilarity among different physiological and pathological magnetospheric states (i.e., pre-storm activity and intense magnetic storms, respectively).

The hourly Disturbance storm-time $\left(D_{s t}\right)$ geomagnetic activity index is computed from an average over 4 mid-latitude magnetic observatories [15], and hence serves as a proxy for the magnetospheric ring current, and thus magnetic storm occurrence. Magnetic storms are the most prominent global phenomenon of geospace dynamics, interlinking the solar wind, magnetosphere, ionosphere, atmosphere and occasionally the Earth's surface [16-18]. Magnetic storms occur when the accumulated input power from the solar wind exceeds a certain threshold.

Herein, we consider one year of $D_{s t}$ data (2001) including two intense magnetic storms, which occurred on 31 March 2001 and 6 November 2001 with minimum $D_{s t}$ values -387 nT and -292 nT respectively, as well as a number of weaker events (e.g., May and August 2001 with $D_{s t} \sim-100 \mathrm{nT}$ in both cases). We employ the time-dependent Tsallis entropy as a measure of dynamical complexity in $D_{s t}$ time series, thus quantifying the degree of predictability in magnetospheric evolution. Our analysis reveals that Tsallis entropy detects the pattern of alterations in $D_{s t}$ time series prior to the intense storm events and is able to discriminate between the different states of the magnetosphere.

\section{Principles of Tsallis Entropy}

The term "entropy" is used in both physics and information theory to describe the amount of uncertainty or information inherent in an object or system. Clausius introduced the notion of entropy into thermodynamics in order to explain the irreversibility of certain physical processes in thermodynamics. In statistical thermodynamics the most general formula for the thermodynamic entropy $S$ of a thermodynamic system is the Boltzmann-Gibbs-Shannon entropy,

$$
S_{B G S}=-k \sum p_{i} \ln p_{i}
$$


where $k$ is the Boltzmann constant and $p_{i}$ are the probabilities associated with the microscopic configurations.

Shannon recognized that a similar approach to Boltzmann-Gibbs-Shannon entropy could be applied to information theory. In his famous 1948 paper [19], he introduced a probabilistic entropy measure $H_{S}$ :

$$
H_{S}(X)=-\sum_{i=1}^{n} p\left(x_{i}\right) \log _{b} p\left(x_{i}\right)
$$

where $b$ is the base of the logarithm used and $p$ denotes the probability mass function of a discrete random variable $X$ with possible values $\left\{x_{1}, \ldots, x_{n}\right\}$.

The aim of statistical mechanics is to establish a direct link between the mechanical laws and classical thermodynamics. One of the crucial properties of the $S_{B G S}$ in the context of classical thermodynamics is extensivity, namely proportionality with the number of elements of the system. The $S_{B G S}$ satisfies this prescription if the subsystems are statistically (quasi-) independent, or typically if the correlations within the system are essentially local. In such cases the system is called extensive.

In general, however, the situation is not of this type and correlations may be far from negligible at all scales. In such cases the $S_{B G S}$ is nonextensive. Tsallis [1,2] introduced an entropic expression characterized by an index $q$ which leads to a nonextensive statistics

$$
S_{q}=k \frac{1}{q-1}\left(1-\sum_{i=1}^{W} p_{i}^{q}\right)
$$

where $p_{i}$ are the probabilities associated with the microscopic configurations, $W$ is their total number and $q$ is a real number. The value of $q$ is a measure of the nonextensivity of the system: $q \rightarrow 1$ corresponds to the standard extensive Boltzmann-Gibbs-Shannon statistics.

This is the basis of the so-called nonextensive statistical mechanics, which generalizes the Boltzmann-Gibbs-Shannon theory. The entropic index $q$ characterizes the degree of nonadditivity reflected in the following pseudo-additivity rule, which is a consequence of Equation (3): $S_{q}(A+B)=$ $S_{q}(A)+S_{q}(B)+(1-q) S_{q}(A) S_{q}(B)$, where $k$ is set to 1 .

The cases $q>1$ and $q<1$ correspond to sub-additivity or super-additivity respectively. For subsystems that have special probability correlations, $S_{B G S}$ corresponds and is the extensive case where $q=1$. Such systems are sometimes referred to as nonextensive [20]. The parameter $q$ itself is not a measure of the complexity of the system but measures the degree of nonextensivity of the system. It is the time variations of the Tsallis entropy for a given $q\left(S_{q}\right)$ that quantify the dynamic changes of the complexity of the system. Lower $S_{q}$ values characterize the portions of the signal with lower complexity.

\section{Calculation of Tsallis Entropy through Symbolic Dynamics}

\subsection{Symbolic Dynamics}

The discovery that simple deterministic systems can show a vast richness of behaviors in response to variations of initial conditions and/or control parameters has been the motivation for an intense interdisciplinary research activity since the 1950s [21-23]. One of the outcomes of this work has been the realization that for an appropriate description of such complex systems, one needs to resort to a 
probabilistic approach [24]. It is well known since the pioneering work of Gibbs and Einstein that we can describe dynamics from two points of view. On the one hand, we have the individual description in terms of trajectories in classical dynamics, or of wave-functions in quantum theory. On the other hand, we have the description in terms of ensembles described by a probability distribution (called the density matrix in quantum theory) [25]. Now, once one leaves the description in terms of trajectories, a basic question that must be dealt with concerns the amount of information one may have access to on the temporal evolution of the system in the course of time.

One of the approaches developed in this context is "coarse-graining", whereby a complex system is viewed as an "information generator" producing messages consisting of a discrete set of symbols defined by partitioning the full continuous phase space into a finite number of cells. We refer to such a description as "symbolic dynamics" [22-24,26]. One of its merits is to provide a link between dynamical systems and information theory $[22,27]$.

From the initial dynamical system we can generate a sequence of symbols, where the dynamics of the original (under analysis) system has been projected. This symbolic sequence can be analyzed by terms of information theory such as entropy estimations, information loss, automaticity and other prominent properties.

There exist some canonical ways for generating symbolic dynamics out of a given dynamical system [22,23,26-28]. To produce symbolic dynamics out of the evolution of a given system, we set up a coarse-grained description incorporating from the very beginning the idea that a physically accessible state corresponds to a finite region rather than to a single point of phase space. Let $C_{i}(i=1,2, \ldots K)$ be the set of cells in phase space constituted by these regions, assumed to be connected and non-overlapping. As time goes on, the phase space trajectory performs transitions between cells thereby generating sequences of $K$-symbols, which may be regarded as the letters of an alphabet. We shall require that, in the course of these transitions, each element of the partition is mapped by the law of evolution on a union of elements.

In this paper, we restrict ourselves to the simplest possible coarse graining of the magnetospheric signal. This is given by choosing a threshold $C$ and assigning the symbols " 1 " and " 0 " to the signal, depending on whether it is above or below the threshold (binary partition). The threshold is usually the mean value of the data considered. In this way, each time window of the original $D_{s t}$ time series for a given threshold is transformed into symbolic sequences, which contains "linguistic" or "symbolic dynamics" characteristics. The selection of a two-symbol alphabet satisfies terms of simplicity and computational convenience.

\subsection{Tsallis Entropy Calculation}

Symbolic dynamics provides a rigorous way of looking at the invariant, robust properties of the dynamics [29]. New methods of nonlinear dynamics derived from the symbolic dynamics have been introduced to distinguish between different states of the system interactions. These methods provide a detailed description and classification of dynamic changes of various real-world time series [30,31].

Herein, we estimate $S_{q}$ based on the concept of symbolic dynamics: from the initial measurements we generate a sequence of symbols, where the dynamics of the original (under analysis) system has been projected [32]. More precisely, the original $D_{s t}$ time series of length $N,\left(X_{1}, X_{2}, \ldots, X_{N}\right)$, is projected 
to a symbolic time series $\left(A_{1}, A_{2}, \ldots, A_{N}\right)$ with $A_{n}$ from a finite alphabet of $\lambda$ letters $(0, \ldots, \lambda-1)$ (see for example [11]).

After symbolization, the next step in the identification of temporal patterns is the construction of symbol sequences with size $L$. We use the technique of lumping. Thus, we stipulate that the symbolic sequence is to be read in terms of distinct successive "blocks" of length $L$, $A_{1}, A_{2}, \ldots, A_{L} / A_{L+1}, \ldots, A_{2 L} / A_{j L+1}, \ldots, A_{(j+1) L}$.

The number of all possible blocks of length $L$ in a $\lambda$-letter alphabet is $N_{\lambda}=\lambda^{L}$. We determine the probabilities of occurrence of each of $N_{\lambda}$ different kind of blocks,

$$
p(L)_{A_{1}, A_{2}, \ldots, A_{L}}=\frac{\text { Number of blocks of the form } \quad A_{1}, A_{2}, \ldots, A_{L} \text { encountered by lumping }}{\text { Total number of blocks encountered by lumping }}
$$

To be more concrete, the simplest possible coarse graining of the $D_{s t}$ index is given by choosing a threshold $C$ (usually the mean value of the data considered) and assigning the symbols " 1 " and " 0 " to the signal, depending on whether it is above or below the threshold (binary partition). Thus, we generate a symbolic time series from a 2-letter $(\lambda=2)$ alphabet $(0,1)$, e.g., $0110100110010110 \ldots$... Reading the sequence by lumping of length $L=2$ one obtains $01 / 10 / 10 / 01 / 10 / 01 / 01 / 10 / \ldots$. The number of all possible kinds of blocks is $\lambda^{L}=2^{2}=4$, namely $00,01,10,11$. Thus, the required probabilities for the estimation of the Tsallis entropy $p_{00}, p_{01}, p_{10}, p_{11}$ are the fractions of the blocks $00,01,10,11$ in the symbolic time series.

The $S_{q}$ for the word length $L$ is

$$
S_{q}(L)=k \frac{1}{q-1}\left(1-\sum_{\left(A_{1}, A_{2}, \ldots, A_{L}\right)}\left[p(L)_{A_{1}, A_{2}, \ldots, A_{L}}\right]^{q}\right)
$$

Broad symbol-sequence frequency distributions produce high entropy values, indicating a low degree of organization. Conversely, when certain sequences exhibit high frequencies, lower entropy values are produced, indicating a high degree of organization.

\section{Results}

A way to examine transient phenomena is to divide their outputting time series into shorter time intervals, related with different activity levels of the corresponding natural systems, and consequently analyze these time windows separately. If this analysis yields different results for time windows associated to an intense magnetic storm, for instance, in comparison to time windows associated to the regular state of the magnetosphere, then a transient behavior can be extracted.

In Figure 1 the $D_{s t}$ time series is presented. The one year $D_{s t}$ data (2001) include 13 major geomagnetic storms, i.e., with $D_{s t} \leq-100 \mathrm{nT}$ (among them 2 storms had "second wave", thus, in total 15 major storms) and 23 moderate storms $\left(-100<D_{s t}<-50 \mathrm{nT}\right)$. The original time series was divided into 5 shorter time series (see triangles denoting 5 distinct time windows in Figure 1). The second and fourth time windows include the $D_{s t}$ variations associated to the 2 intense magnetic storms of 31/3/2001 and 6/11/2001, respectively. Within each of the 5 time windows, the Tsallis entropy $S_{q}$ is calculated for different values of the entropic index $q(1,1.2,1.5,1.76,1.84,2,2.5,3,4,5)$ using the technique of lumping. 
Figure 1. From top to bottom are shown $D_{s t}$ time series along with time variations of Shannon entropies and Tsallis entropies, $S_{q}$. Tsallis entropies were derived using a $q$ parameter value of 1.84. The 31 March and 6 November 2001 magnetic storms are marked with red. The triangles denote the time intervals corresponding to the five time windows used for the calculations presented in Figure 2. The red dashed line in the $S_{q}$ plot marks a possible boundary value ( 0.7$)$ for the transition of the system to the lower complexity, which is characteristic of the different state of the magnetosphere.
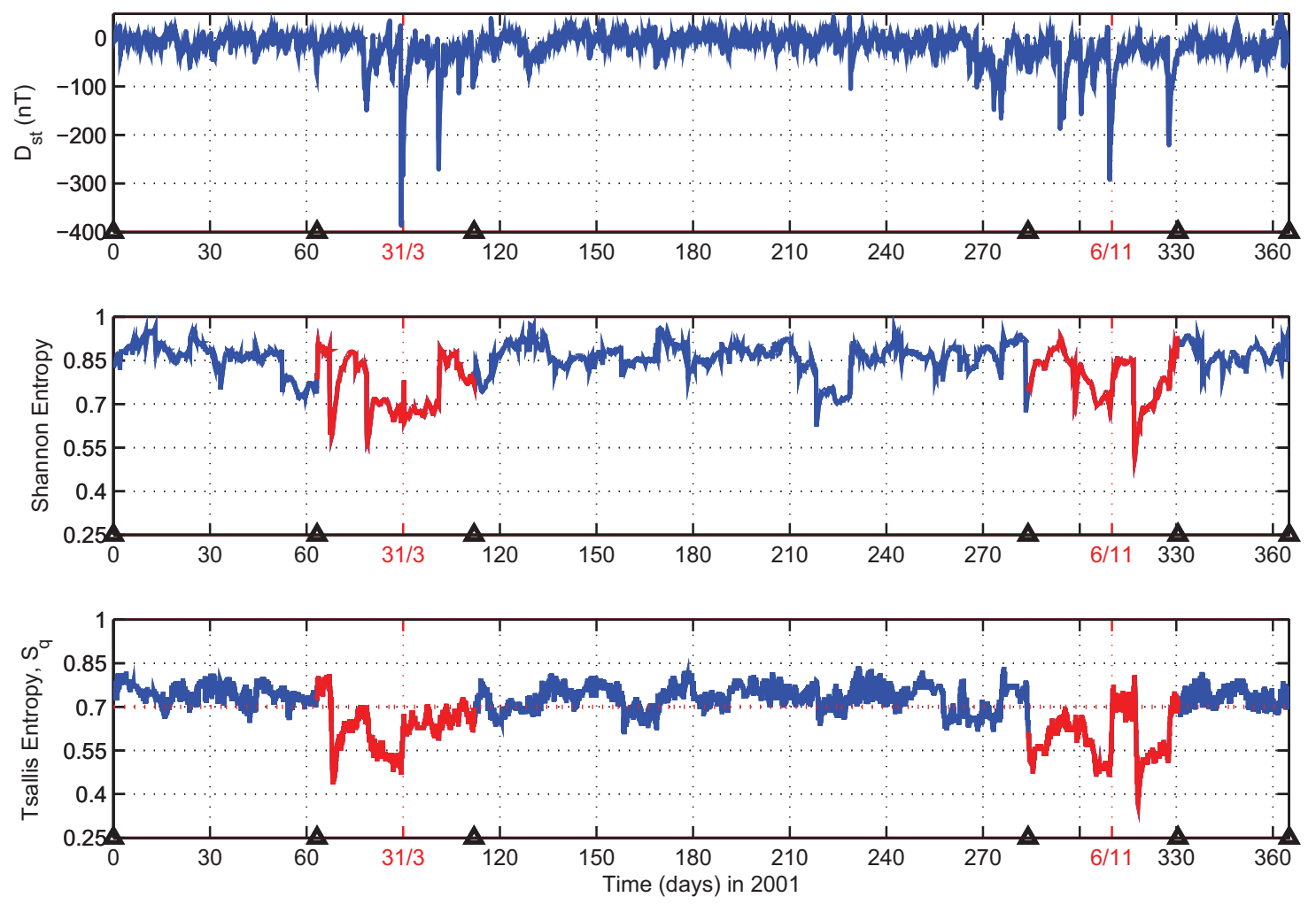

Figure 2 shows the normalized $S_{q}$ values for the 5 different windows. The entropies have been normalized with respect to the entropies given in Equation (5) for a uniform distribution of probabilities. We study the temporal evolution of the $S_{q}$ as the global instability is approaching. Time windows 1, 3 and 5 are referred to the normal state of magnetosphere. Their $S_{q}$ values are lower in respect to that given in Equation (5) for a uniform distribution of probabilities. This evidence indicates the existence of an organization in the magnetosphere even in this normal state. The entropies in windows 2 and 4 drop to rather significantly lower values suggesting the appearance of a new distinct state in the magnetosphere, which is characterized by a lower complexity in comparison to that of the normal epoch of the magnetosphere. This is the case for all $q \mathrm{~s}$.

As expected, our results depend upon the Tsallis $q$ value. Figure 2 clearly illustrates the superiority of the $q$ values restricted in the range $1<q<2$ to magnify differences of the $S_{q}$ and thus of the complexity as the global instability is approaching. It is worth mentioning that the nonextensive $q$ parameters that clearly quantify the temporal evolution of the complexity in the $D_{s t}$ time series are in full agreement with the upper limit $q<2$ obtained from several studies involving the Tsallis nonextensive framework ([33] and references therein). Moreover, they are in harmony with an underlying sub-extensive system, $q>1$, 
verifying the emergence of strong interactions in the magnetosphere, especially during the occurrence of an intense magnetic storm.

Figure 2. The normalized Tsallis entropies $S_{q}$ calculated at the five time windows, derived after the initial $D_{s t}$ time series was divided into five shorter time intervals as shown in Figure 1, for ten different values of the entropic index $q(q=$ $[1,1.2,1.5,1.76,1.84,2,2.5,3,4,5])$. On abscissa it is noted the central day of each time window. The arrows point to the Tsallis entropies corresponding to $q=1.84$.

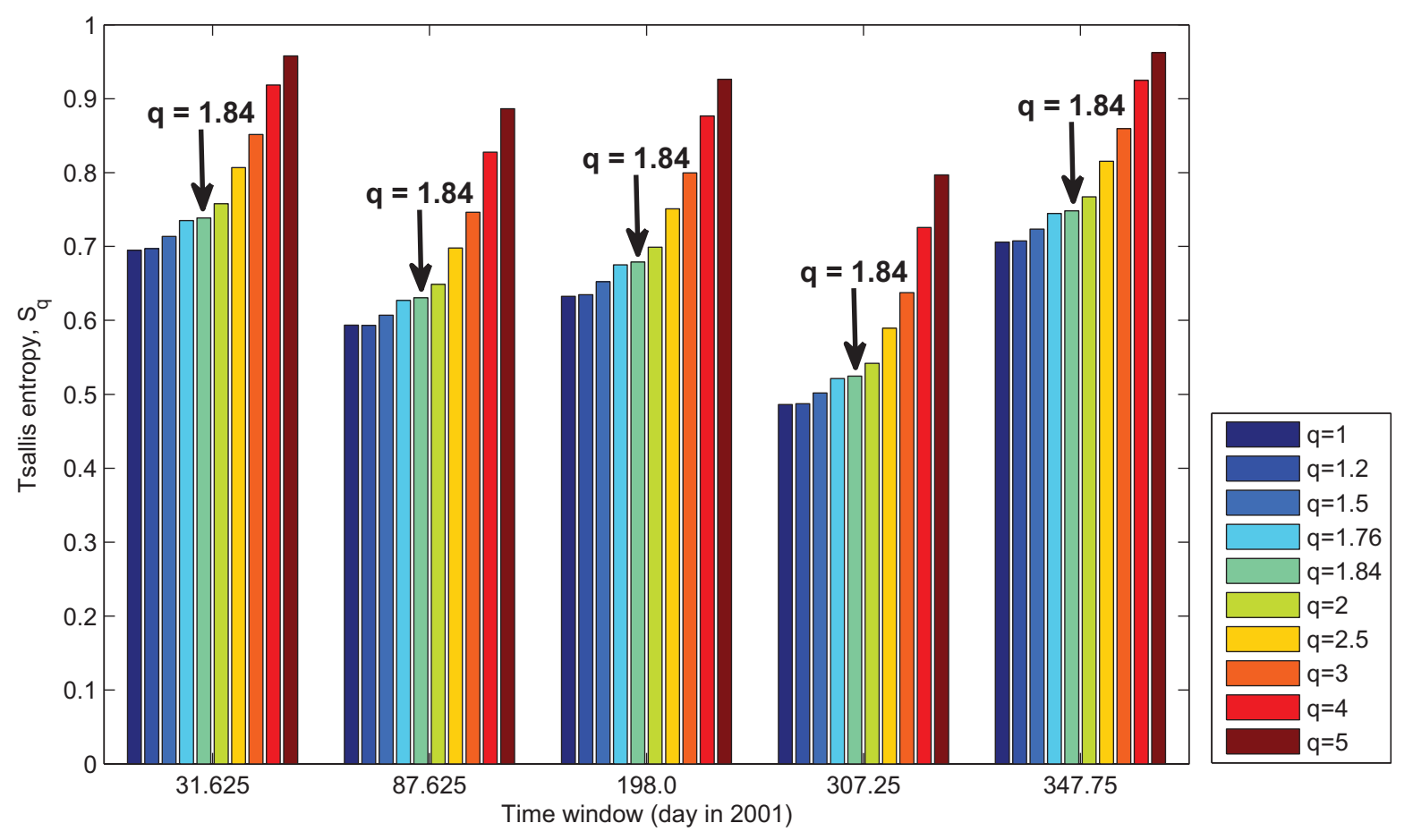

It is worth mentioning that the case $q=1$ (Figure 2) seems to provide a hint for two different patterns in the evolution of the system under study. However, the magnetosphere, due to the appearance of strong interactions across its system, especially immediate before the magnetic storm occurrence, clearly violates the Boltzmann-Gibbs-Shannon statistics. Therefore the result for this particular case has absolutely no physical meaning.

As mentioned, the results depend upon the entropic index $q$. Therefore the appropriate choice of the $q$ index is significant and needs to be examined [34]. It is expected that, for every specific system, better discrimination will be achieved with appropriates ranges of $q$ values [3]. Thus, a challenge will be to estimate the appropriate value of $q$ which is associated with the generation of magnetic storms. We attempt an estimation of the appropriate value of the $q$ index based on ideas rooted in the areas of scale invariance and universality [14] in Section 5.

Various tests have been performed with different lengths of word $(L)$ for the purposes of symbolic dynamics analysis (see Section 3.2), as well as with different candidate lengths of time windows of the initial time series. We managed to achieve, by gradually varying both kinds of length, the optimal values in order to best describe and resolve the transition from the normal magnetospheric state to intense magnetic storms. Because of space limitations, only the results for the optimal length values are given in this paper, which are also the most interesting from the physical point of view. 


\section{Estimation of the Appropriate Entropic Index $q$}

Different values of the entropic index $q$ lead to different Tsallis entropies $S_{q}$. Therefore, a crucial question is now arising. How is the appropriate value for the entropic index $q$ calculated in order to derive optimum Tsallis entropies $S_{q}$ for our time series with respect to better characterization of the state of the system under study?

Empirical evidence has been mounting that supports the possibility that a number of systems arising in disciplines as diverse as physics, biology, engineering, and economics may have certain quantitative features that are intriguingly similar. These properties can be conveniently grouped under the headings of "scale invariance" and "universality" [35].

A model for earthquake dynamics coming from a nonextensive Tsallis formalism, starting from first principles, has been recently introduced by Sotolongo-Costa and Posadas [36]. Based on this model, an energy distribution function, which gives the Guttenberg-Richter empirical law [37] as a particular case, has been analytically deduced in the framework of Tsallis statistical mechanics (see Equation (8) in [36]). Their equation provides an excellent fit to seismicities generated in various large geographic areas usually identified as seismic regions. Silva et al. [38] have subsequent revised this model considering the current definition of the mean value, i.e., the so-called $q$-expectation value. They also suggested an energy distribution function that also provides an excellent fit to seismicities

$$
\log \left(N_{>m}\right)=\log N+\left(\frac{2-q}{1-q}\right) \log \left[1-\left(\frac{1-q}{2-q}\right)\left(\frac{10^{2 m}}{\alpha^{2 / 3}}\right)\right]
$$

where $N$ is the total number of earthquakes, $N_{>m}$ the number of earthquakes with magnitude larger than $m$, and $m \approx \log \varepsilon . \alpha$ is the constant of proportionality between the earthquake energy, $\varepsilon$, and the size of fragment, $r\left(\varepsilon \sim r^{3}\right)$. Importantly, the associated $q$-values with the aforementioned Gutenberg-Richter type law [Equation (6)] for 3 different regions (faults) in the world and by considering a threshold $(\mathrm{m}$ ) equal to earthquake magnitude 3 are 1.6, 1.63 and 1.71, respectively.

The primary question we can ask in the context of complex systems theory is whether the aforementioned nonextensive laws not only successfully describe the magnitude distribution of earthquakes in the Earth but also magnetic storms occurred in the terrestrial magnetosphere [13,14]. We note that this model for earthquake generation mechanism was also applied successfully to pre-seismic electromagnetic emissions [39,40] and just recently to Southern California seismicity [41].

We now examine whether the energy distribution function (Equation 6) corresponding to a nonextensive Tsallis statistics is able to describe the $D_{s t}$ time series. Figure 3 shows that Equation (6) provides an excellent fit to the experimental data, incorporating the characteristics of nonextensivity statistics into the distribution of the magnetospheric events. Herein, $N$ is the total number of $D_{s t}$ data, $N_{>m}$ the number of $D_{s t}$ values with magnitude larger than $m, G_{>m}=N_{>m} / N$ the relative cumulative number of events with magnitude larger than $m$, and $\alpha$ a proportionality constant. The magnitude $m$ is approximately $\log \varepsilon$, where $\varepsilon$ is the squared $D_{s t}$ value. (In the case of $D_{s t}$ index the square of the amplitude of the magnetic field is proportional to energy). The best-fit for this analysis is given by a $q$ parameter value equal (within a confidence limit of 95\%) to 1.84 , whereas the threshold $m$ is taken $-30 \mathrm{nT}$. (The associated with the model parameters minimize the $\chi^{2}$ merit function given by the sum of the squared residuals.) 
Figure 3. We use the Gutenberg-Richter (G-R) type law for the nonextensive Tsallis statistics (Equation 6) to calculate the relative cumulative number of $D_{s t}$ data, $N(>m) / N$ (upper panel). There is an excellent agreement of the aforementioned formula with the $D_{s t}$ time series. The threshold is $-30 \mathrm{nT}$ which results in 164 events, and the associated Tsallis entropic parameter is $q=1.84$.

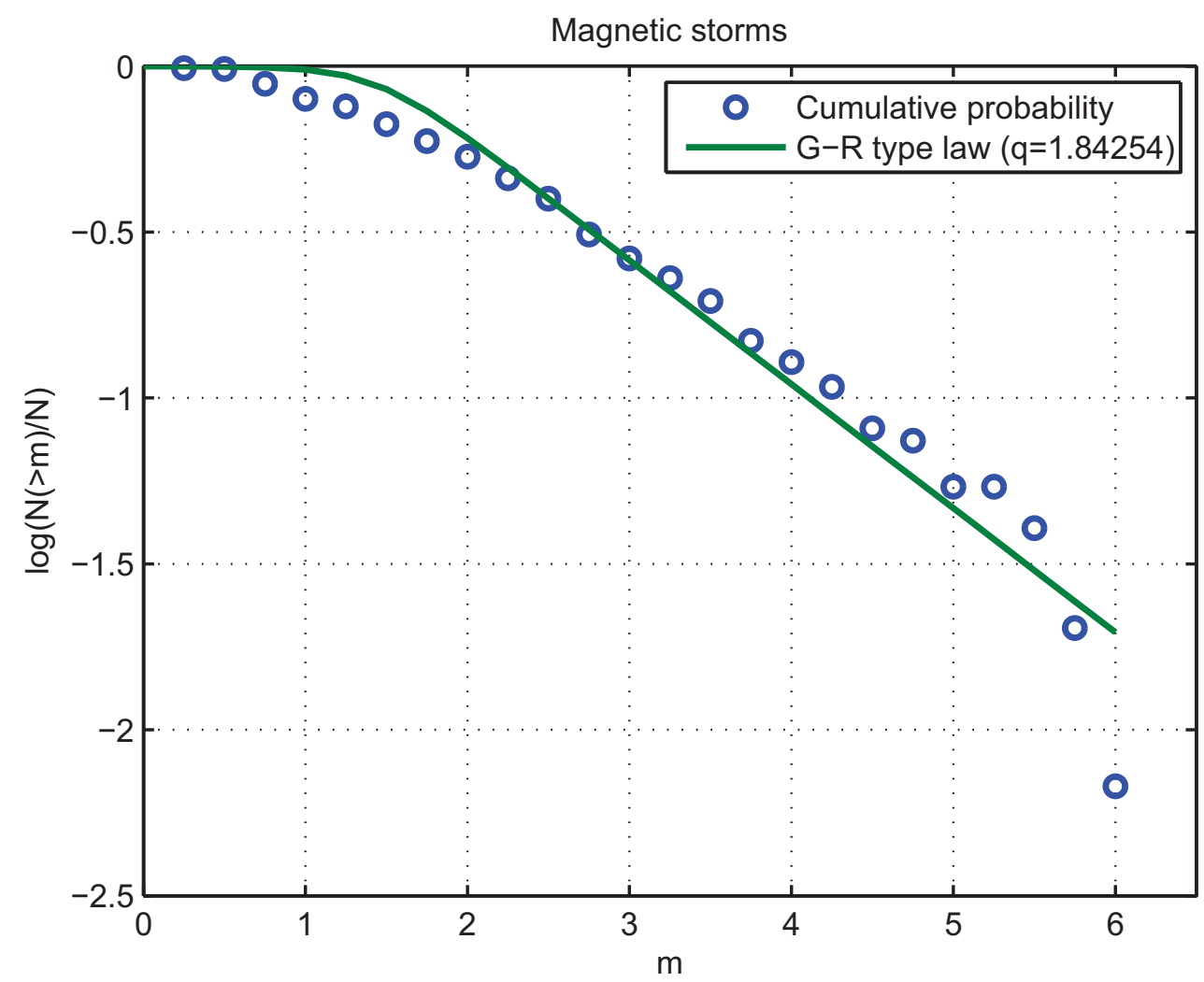

We clarify that we consider a sequence of $N$ consecutive values that surpass the threshold to constitute an "event" and measure its energy by integrating (summing) the individual data-point energies that comprise it. Though this method better captures the system dynamics, it tends to unify near-concurrent events when they both exceed the corresponding threshold, thus attributing lower probabilities to very energetic events. This "merging" explains the divergence from the theoretical curve at the far right of the graph in the lower part of the curve in Figure 3.

Figure 1 shows the temporal evolution of Shannon and Tsallis entropy for the $D_{s t}$ time series. The calculation of Tsallis entropy values is based on the use of Tsallis parameter $q$ found when applying Equation (6) to the data, i.e., 1.84. Figure 1 shows that generally in time intervals around extreme magnetospheric events (i.e., intense magnetic storms of 31/3/2001 and 6/11/2001) the corresponding Tsallis entropies of $D_{s t}$ data attain lower values than for the rest of the time series. Lower entropy means lower complexity or a higher degree of organization for the corresponding natural system (i.e., magnetosphere) around the particular extreme event.

Figure 1 further demonstrates that Tsallis entropy yields superior results in comparison to Shannon entropy regarding the detection of dynamical complexity in the Earth's magnetosphere (i.e., offer a clearer picture of the transition from normal state to magnetic storms). A possible explanation for this is that Tsallis is an entropy obeying a nonextensive statistical theory, which is different from the usual 
Boltzmann-Gibbs-Shannon statistical mechanics obeyed by Shannon entropy. Therefore, it is expected to better describe the dynamics of the magnetosphere, which is a nonequilibrium physical system with large variability. Next, we further demonstrate the universality of Equation (6) by showing its applicability to other extreme events of the solar-terrestrial system as in the case of solar flares, for instance [14].

\section{Nonextensive Tsallis Analysis of Solar Flares}

Solar flares are highly energetic explosions from active regions of the Sun in the form of EM radiation, particle and plasma flows powered by strong and twisted magnetic fields. A solar flare occurs when magnetic energy that has built up in the solar atmosphere is suddenly released. In particular, radiation is emitted across virtually the entire EM spectrum, from radio waves at the long-wavelength end, through optical emission to X-rays and $\gamma$-rays at the short-wavelength end.

The X-ray flux data presented in Figure 4 (upper panel) include $6 \mathrm{X}$-class, $27 \mathrm{M}$-class, $138 \mathrm{C}$-class and 197 B-class solar flares that took place from 1 December 2004 to 28 February 2005 (for an event list see [42]). In particular, a series of $\mathrm{M}$ - and X-class flares occurred in the single extensive active region AR0720 between 10 and 23 January 2005. Moreover, between January 15th and 19th, this sunspot produced four powerful solar flares. When it exploded a fifth time on January 20th released the highest concentration of protons ever directly measured, taking only 15 minutes after observation to reach Earth, indicating a velocity of approximately one-half light speed.

We now examine whether the Tsallis-based energy distribution function [Equation (6)] is also able to describe the X-ray fluxes. Figure 4 (lower panel) shows that Equation (6) provides an excellent fit to the experimental data, incorporating the characteristics of nonextensivity statistics into the distribution of the solar events. Herein, $N$ is the total number of X-ray fluxes, $N_{>m}$ the number of X-ray flux values with magnitude larger than $m, G_{>m}=N_{>m} / N$ the relative cumulative number of events with magnitude larger than $m$, and $\alpha$ a proportionality constant. The magnitude $m$ is approximately $l o g \varepsilon$, where $\varepsilon$ is the integrated X-ray flux value. (In the case of X-ray flux we take the integral of fluxes for calculating energy). The best-fit for this analysis is given by a $q$ parameter value equal (within a confidence limit of $95 \%$ ) to 1.82 , whereas the threshold $m$ is taken $10^{-6} \mathrm{~W} / \mathrm{m}^{2}$.

The aforementioned result indicates that solar flares and magnetic storms obey nonextensive laws which are scale invariant, and that these laws are universal in the sense that they do no depend on details concerning the actual species. The aforementioned finding could be considered as a further indication of the universality of fractal properties among a large number of various geophysical processes. This is not a surprising result. Previously, de Arcangelis et al. [43] also demonstrated that the stochastic processes underlying apparently different phenomena such as solar flares and earthquakes have universal properties. 
Figure 4. GOES-12 5-minute averages X-ray flux, $X_{l}$ (1-8 Angstrom) time series (upper panel). The 20 January 2005 solar flare is marked with red. We use the Gutenberg-Richter (G-R) type law for the nonextensive Tsallis statistics (Equation 6) to calculate the relative cumulative number of X-ray flux data, $N(>m) / N$ (lower panel). There is an excellent agreement of the aforementioned formula with the X-ray flux time series. The threshold is $10^{-6} \mathrm{~W} / \mathrm{m}^{2}$ which results in 141 events, and the associated parameter is $q=1.82$.
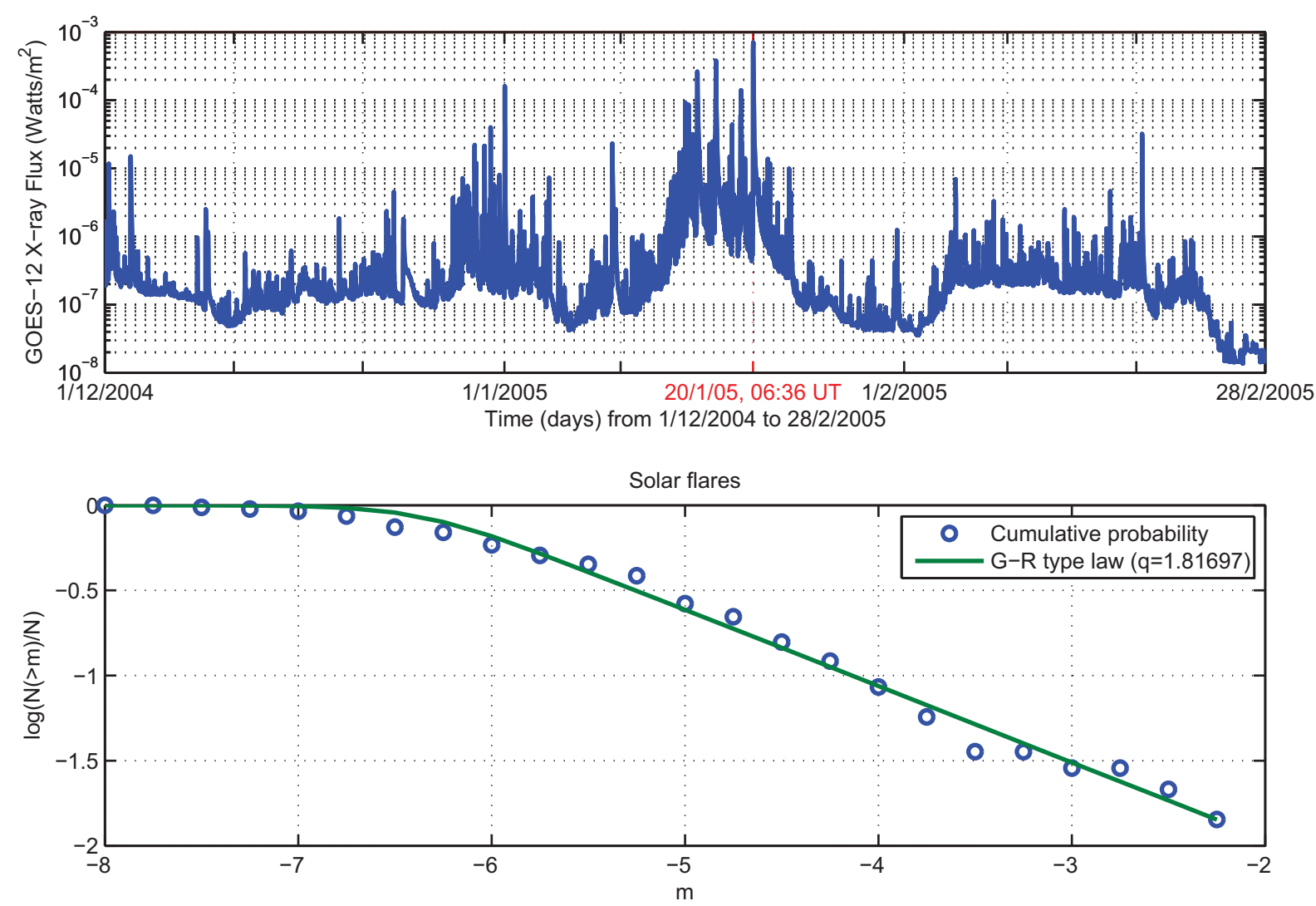

\section{Conclusions and Discussion}

Accumulated evidence points to the complex character of magnetosphere dynamics. For instance, Baker et al. [57], Vassiliadis et al. [45] and Sharma et al. [46] studied the occurrence of low dimensional chaos in magnetospheric activity, while Klimas et al. [47] discussed the nonlinear character of magnetosphere dynamics. Other studies $[48,49]$ witnessed several aspects of the complex character of the plasma sheet. Thus, recent advances in the study of complexity and complex systems open new research perspectives to the investigation of the magnetospheric dynamics [50].

Our analysis reveals that Tsallis entropy detects the pattern of alterations in $D_{s t}$ time series prior to the intense storm events and is able to discriminate between the different states of the magnetosphere. The Tsallis entropy sensitively shows the complexity dissimilarity among different "physiological" (normal) and "pathological" states (intense magnetic storms). The Tsallis entropy implies the emergence of two distinct patterns: (i) a pattern associated with the intense magnetic storms, which is characterized by a higher degree of organization, and (ii) a pattern associated with normal periods, which is characterized by a lower degree of organization. 
In a series of papers, examining the applicability of various information measures to investigate dynamical complexity in the magnetosphere, it has been found that the application of Tsallis entropy to the analysis of the $D_{s t}$ time series yields superior results for detecting dynamical complexity changes associated with magnetic storms in comparison to other entropy measures [12,51,52].

Magnetic storms and solar flares follow the same Tsallis-based energy distribution function previously derived for earthquakes. Thus, the property of universality in solar flare, magnetic storm and earthquake dynamics arise naturally in the framework of Tsallis statistical mechanics [14].

The conditions on the Sun and in the solar wind, magnetosphere, ionosphere, and thermosphere that can influence the performance and reliability of space-borne and ground-based technological systems and endanger human life or health are termed as space weather. Space weather research addresses a large number of physical processes in space, ranging from solar activity to its influence on interplanetary space and geospace and its effects encountered at the surface of the Earth. Physical understanding of this chain is based on a combination of observations, data analysis and interpretation, and theoretical and empirical modeling [53].

Accurate detection of the dissimilarity of complexity between normal and abnormal states (e.g., pre-storm activity and magnetic storms) can vastly improve space weather diagnosis and, consequently, the mitigation of space weather hazards. Moreover, the convergence of the results presented in this paper from the application of nonextensive Tsallis entropy to the $D_{s t}$ time series with other information measures [11,12,52,54,55] can potentially increase the reliability of forecasting techniques and can therefore improve space weather forecasting and modeling. For instance, the application of Hurst exponent, $H$, analysis [52,54] to the $D_{s t}$ time series showed the existence of two different patterns: (i) a pattern associated with the intense magnetic storms, which is characterized by a fractional Brownian persistent behavior $(0.5<H<1)$; (ii) a pattern associated with normal periods, which is characterized by a fractional Brownian anti-persistent behavior $(0<H<0.5)$. The anti-persistent time windows correspond to the time windows of high Tsallis entropies, while the persistent time windows correspond to the time windows of low Tsallis entropies. It is worth noting that Balasis et al. [55] recently applied the typical formula for log-periodicity in time, which was previously used for examining log-periodicity observed in the seismic precursors of the 1989 Loma Prieta earthquake [56], to the $D_{s t}$ time series and achieved a successful prediction of a major magnetic storm. This is another example of transferring ideas from seismology to magnetospheric physics, similar to the use of Equation (6), which was originally derived to describe earthquake dynamics in the framework of Tsallis statistical mechanics, for fitting the $D_{s t}$ data as presented in this paper.

Herein, we also show results from the application of nonextensive Tsallis statistics to solar flare events. Solar flares have been a field of intense complexity studies since 2 decades ago. In the late 1980s, Bak et al. [57] introduced the concept of self-organized criticality (SOC) in order to describe slowly-driven nonequilibrium complex systems with extended degrees of freedom and a high level of nonlinearity. In the early 1990s, Lu and Hamilton [58] were the first to applied SOC theory to solar flares. They suggested that the behavior of the coronal magnetic field corresponds to that of a conservative sandpile model where the random twisting of the solar magnetic field by the photospheric convective motion plays the part of the addition of sand grains. Next, Georgoulis et al. [59] showed that cellular 
automata SOC models are able to reproduce efficiently most of the statistical properties of solar flares, at least those seen in hard X-ray wavelengths.

Hamon et al. [60] applied an established SOC model for earthquakes, i.e., the Olami-Feder-Christensen (OFC) model [61], to the problem of solar flares. They showed that the probability density of event magnitudes given by the OFC model was in good agreement with most of the flare observations and provided evidence of correlation in time between flares in the OFC model. They found that the system, which is usually not in a SOC state, can be described as being on the edge of SOC. The critical features of the system depend on the system bulk dissipation and driving rates, and, thus, they suggested that the concept of universality must be used with care. However, they also pointed out that the driven model shows many similarities and correspondences with observations from the physics of stellar flares suggesting they may be considered a manifestation of general behaviors of complex system dynamics. In this context, it is not surprising the fact that the Tsallis-based energy distribution function, previously derived to describe earthquake dynamics, is also applicable to the field of solar flare dynamics, as suggested here. The SOC and OFC models previously applied to fit solar flare data by Lu and Hamilton [58] and Hamon et al. [60], respectively, fit the data at least as well as the nonextensive model fits the X-ray flux data in this paper.

The universal character of various extreme events and associated critical phenomena is an outstanding scientific challenge. Transferring ideas, concepts, and results between investigators from hitherto disparate areas will cross-fertilize and lead to important new results. The evidence of a universal statistical behavior suggests the possibility of a common approach to forecasting of space weather and earthquakes [14]. Thus, the transfer of ideas and methods of seismic forecasting to the prediction of solar flares and magnetic storms could improve space weather forecasting. In a similar way, a better comparison and possibly combination of results with other well-established models $[58,60]$ associated with extreme space events will be gained, which is of paramount importance for understanding the emergence of criticality in disparate areas of research such as geophysics, space and solar physics.

\section{Acknowledgements}

We would like to thank the referees of this paper for constructive comments and remarks. The $D_{s t}$ data are provided by the World Data Center for Geomagnetism, Kyoto [62]. The X-rays flux data are downloaded from the Space Physics Interactive Data Resource of the National Geophysical Data Center [63].

\section{References}

1. Tsallis, C. Possible generalization of Boltzmann-Gibbs statistics. J. Stat. Phys. 1988, 52, 479-487.

2. Tsallis, C. Generalized entropy-based criterion for consistent testing. Phys. Rev. E 1998, 58, 1442-1445.

3. Tsallis, C. Introduction to Nonextensive Statistical Mechanics, Approaching a Complex Word; Springer: New York, NY, USA, 2009; p. 382. 
4. Group of Statistical Physics, CBPF. Verifications and applications (a selection): Experimental, observational and computational. Available online: http://tsallis.cat.cbpf.br/experimental.htm (accessed on 28 September 2011).

5. Group of Statistical Physics, CBPF. Verifications and applications (a selection): Theoretical. Available online: http://tsallis.cat.cbpf.br-theoretical.htm (accessed on 28 September 2011).

6. Group of Statistical Physics, CBPF. Nonextensive statistical mechanics and thermodynamics: Bibliography. Available online: http://tsallis.cat.cbpf-.br/TEMUCO.pdf (accessed on 28 September 2011).

7. Burlaga, L.F.; Vinas, A.F. Triangle for the entropic index q of non-extensive statistical mechanics observed by Voyager 1 in the distant heliosphere. Physica A 2005, 356, 375-384.

8. Burlaga, L.F.; Ness, N.F. Compressible "turbulence" observed in the heliosheath by Voyager 2. Astrophys. J. 2009, 703, 311-324.

9. Leubner, M.P.; Vörös, Z. A nonextensive entropy approach to solar wind intermittency. Astrophys. J. 2005, 618, 547-555.

10. Leubner, M.P.; Vörös, Z. A nonextensive entropy path to probability distributions in solar wind turbulence. Nonlin. Process. Geophys. 2005, 12, 171-180.

11. Balasis, G.; Daglis, I.A.; Papadimitriou, C.; Kalimeri, M.; Anastasiadis, A.; Eftaxias, K. Dynamical complexity in Dst time series using nonextensive Tsallis entropy. Geophys. Res. Lett. 2008, 35, L14102.

12. Balasis, G.; Daglis, I.A.; Papadimitriou, C.; Kalimeri, M.; Anastasiadis, A.; Eftaxias, K. Investigating dynamical complexity in the magnetosphere using various entropy measures. J. Geophys. Res. 2009, 114, A00D06.

13. Balasis, G.; Eftaxias, K. A study of non-extensivity in the Earth's magnetosphere. Eur. Phys. J.-Spec. Top. 2009, 174, 219-225.

14. Balasis, G.; Daglis, I.A.; Anastasiadis, A.; Papadimitriou, C.; Mandea, M.; Eftaxias, K. Universality in solar flare, magnetic storm and earthquake dynamics using Tsallis statistical mechanics. Physica A 2011, 390, 341-346.

15. World Data Center for Geomagnetism, Kyoto. Available online: http://swdcwww.kugi.kyoto-u.ac. jp/ (accessed on 28 September 2011).

16. Daglis, I.A.; Kozyra, J.U. Outstanding issues of ring current dynamics. J. Atmos. Solar-Terr. Phys. 2002, 64, 253.

17. Daglis, I.A.; Kozyra, J.U.; Kamide, Y.; Vassiliadis, D.; Sharma, A. S.; Liemohn, M. W.; Gonzalez, W. D.; Tsurutani, B. T.; Lu, G. Intense space storms: Critical issues and open disputes. J. Geophys. Res. 2003, 108, 1208.

18. Daglis, I.A.; Balasis, G.; Ganushkina, N.; Metallinou, F.-A.; Palmroth, M.; Pirjola, R.; Tsagouri, I.A. Investigating dynamic coupling in geospace through the combined use of modeling, simulations and data analysis. Acta Geophys. 2009, 57, 141.

19. Shannon, C.E. A mathematical theory of communication. Bell Syst. Tech. J. 1948, 27, 379-423, 623-656.

20. Boon, J.; Tsallis, C. Special issue overview nonextensive statistical mechanics: New trends, new perspectives. Europhys. News 2005, 36, 185-186. 
21. Khinchin, A.I. Mathematical Foundations of Information Theory; Dover: New York, NY, USA, 1957; p. 120.

22. Nicolis, J.S. Chaos and Information Processing; World Scientific: Singapore, 1991; p. 304.

23. Nicolis, G. Introduction to Nonlinear Science; Cambridge University Press: Cambridge, UK, 1995; p. 254.

24. Nicolis, G.; Gaspard P. Toward a probabilistic approach to complex systems. Chaos Soliton. Fractal. 1994, 4, 41-57.

25. Prigogine, I.; Driebe, D.J. Time, chaos and the laws of nature. In Nonlinear Dynamics, Chaotic and Complex Systems; Infeld, E., Zelazny, R., Galkowski, A., Eds.; Cambridge University Press: Cambridge, UK, 1997; pp. 206-224.

26. Nicolis, G.; Nicolis, C.; Nicolis, J.S. Chaotic dynamics, Markov partitions and Zipf's law. J. Stat. Phys. 1989, 54, 915-924.

27. Ebeling, W.; Nicolis, G. Word frequency and entropy of symbolic sequences: A dynamical Perspective. Chaos Soliton. Fractal. 1992, 2, 635-650.

28. Nicolis, G.; Rao, G.; Rao, J.; Nicolis, C. Generation of spatially asymmetric, information-rich structures in far from equilibrium systems. In Coherence and Chaos in Dynamical Systems; Christiansen, P.L., Parmentier, R.D., Eds.; Manchester University Press: Manchester, UK, 1988.

29. Bailin, H. Elementary Symbolic Dynamics and Chaos in Dissipative Systems; World Scientific: Singapore, 1989; p. 476.

30. Schwarz, U.; Arnold, O.B.; Kurths, J.; Witt, A. Analysis of solar spike events by means of symbolic dynamics methods. Astron. Astrophys. 1993, 277, 215-224.

31. Wanliss, J.A.; Anh, V.V.; Yu, Z.-G.; Watson, S. Multifractal modeling of magnetic storms via symbolic dynamics analysis. J. Geophys. Res. 2005, 110, A08214.

32. Bailin, H. Symbolic dynamics and characterization of complexity. Physica D 1991, 51, 161-176.

33. Vilar, C.; Franca, G.; Silva, R.; Alcaniz, J. Nonextensivity in geological faults? Physica A 2007, 377, 285-290.

34. Naudts, J. Deformed exponentials and logarithms in generalized thermostatistics. Physica A 2002, 316, 323-334.

35. Stanley H.E. Scaling, universality, and renormalization: Three pillars of modern critical phenomena. Rev. Mod. Phys. 1999, 71, 358-366.

36. Sotolongo-Costa, O.; Posadas, A. Fragment-asperity interaction model for earthquakes. Phys. Rev. Lett. 2004, 92, 048501.

37. Guttenberg, B.; Richter, C.F. Frequency of earthquakes in California. Bull. Seismol. Soc. Am. 1944, 34, 185-188.

38. Silva, R.; Franca, G.; Vilar, C.; Alcaniz, J. Nonextensive models for earthquakes. Phys. Rev. E 2006, 3, 026102.

39. Kalimeri, M.; Papadimitriou, C.; Balasis, G.; Eftaxias, K. Dynamical complexity detection in pre-seismic emissions using nonadditive Tsallis entropy. Physica A 2008, 387, 1161-1172.

40. Papadimitriou, C.; Kalimeri, M.; Eftaxias, K. Nonextensivity and universality in the earthquake preparation process. Phys. Rev. E. 2008, 77, 036101. 
41. Telesca, L. Tsallis-based nonextensive analysis of the southern California seismicity. Entropy 2011, 13, 1267-1280.

42. NOAA/NWS, Space Weather Prediction Center. Available online: http://www.swpc.noaa.gov/ (accessed on 28 September 2011).

43. de Arcangelis, L.; Godano, C.; Lippiello, E.; Nicodemi, M. Universality in solar flare and earthquake occurrence. Phys. Rev. Lett. 2006, 96, 051102.

44. Baker, D.; Klimas, A.; McPherron, R.; Buechner, J. The evolution from weak to strong geomagnetic activity: An interpretation in terms of deterministic chaos. Geophys. Res. Lett. 1990, 17, 41.

45. Vassiliadis, D.; Sharma, A.S.; Eastman, T.E.; Papadopoulos, K. Low-dimensional chaos in magnetospheric activity from AE time series. Geophys. Res. Lett. 1990, 17, 1841.

46. Sharma, A.S.; Vassiliadis, D.; Papadopoulos, K. Reconstruction of low-dimensional magnetospheric dynamics by singular spectrum analysis. Geophys. Res. Lett. 1993, 20, 335.

47. Klimas, A.J.; Vassiliadis, D.; Baker, D.N.; Roberts, D.A. The organized nonlinear dynamics of the magnetosphere. J. Geophys. Res. 1996, 101, 13089.

48. Angelopoulos, V.; Mukai, T.; Kokubun, S. Evidence for intermittency in Earth's plasma sheet and implications for self-organized criticality. Phys. Plasmas 1999, 6, 4161.

49. Lui, A.T.Y. Physics of Space Plasmas; Chang, T., Jasperse, J.R., Eds.; MIT Geo/Cosmo Plasma Physics: Cambridge, MA, USA, 1998; Volume 15, p. 233.

50. Consolini, G. Complexity in the Earth's magnetospheric dynamics. Ann. Geophys. 2003, 21, 1911.

51. Balasis, G.; Daglis, I.A.; Anastasiadis, A.; Eftaxias, K. Investigating magnetospheric dynamics using various complexity measures. In Modern Challenges in Nonlinear Plasma Physics: A Festschrift Honoring the Career of Dennis Papadopoulos; Vassiliadis, D., Fung, S.F., Shao, X., Daglis, I.A., Huba, J.D., Eds.; American Institute of Physics: Melville, NY, USA, 2010; AIP Conference Proceedings 1320, pp. 65-71, doi:10.1063/1.3544339.

52. Balasis, G.; Daglis, I.A.; Anastasiadis, A.; Eftaxias, K. Detection of dynamical complexity changes in Dst time series using entropy concepts and rescaled range analysis. In The Dynamic Magnetosphere, IAGA Special Sopron Book Series; Liu, W., Fujimoto, M., Eds.; Springer: Dordrecht, The Netherlands, 2011; Volume 3, Part 3, pp. 211-220, doi: 10.1007/978-94-007-0501-2_12.

53. Watermann, J.; Wintoft, P.; Sanahuja, B.; Saiz, E.; Poedts, S.; Palmroth, M.; Milillo, A.; Metallinou, F.-A.; Jacobs, C.; Ganushkina, N.Y.; et al. Models of the solar wind structures and its interaction with the Earth's space environment. Space Sci. Rev. 2009, 147, 233-270.

54. Balasis, G.; Daglis, I.A.; Kapiris, P.; Mandea, M.; Vassiliadis, D.; Eftaxias, K. From pre-storm activity to magnetic storms: A transition described in terms of fractal dynamics. Ann. Geophys. 2006, 24, 3557-3567.

55. Balasis, G.; Papadimitriou, C.; Daglis, I.A.; Anastasiadis, A.; Athanasopoulou, L.; Eftaxias, K. Signatures of discrete scale invariance in Dst time series. Geophys. Res. Lett. 2011, 38, L13103.

56. Huang, Y.; Saleur, H.; Sornette, D. Reexamination of log-periodicity observed in the seismic precursors of the 1989 Loma Prieta earthquake. J. Geophys. Res. 2000, 105, doi:10.1029/2000JB900308. 
57. Bak, P.; Tang, C.; Wiesenfeld, K. Self-organized criticality? An explanation of 1/f noise. Phys. Rev. Lett. 1987, 59, 381.

58. Lu, E.T.; Hamilton, R.J. Avalanches and distribution of solar flares. Astrophys. J. 1991, 380, L89-L92.

59. Georgoulis, M.K.; Vilmer, N.; Crosby, N.B. A comparison between statistical properties of solar X-ray flares and avalanche predictions in cellular automata statistical flare models. Astron. Astrophys. 2001, 367, 326-338.

60. Hamon, D.; Nicodemi, M.; Jensen, H.J. Continuously driven OFC: A simple model of solar flare statistics. Astron. Astrophys. 2002, 387, 326-334.

61. Olami, Z.; Feder, H.J.S.; Christensen, K. Self-organized criticality in a continuous, nonconservative cellular automaton modeling earthquakes. Phys. Rev. Lett. 1992, 68, 1244-1248.

62. Geomagnetic Equatorial Dst Index Home Page. Available online: http://wdc.kugi.kyoto-u.ac.jp/ dstdir/index.html (accessed on 28 September 2011).

63. The Space Physics Interactive Data Resource (SPIDR). Available online: http://spidr.ngdc.noaa. gov/spidr/ (accessed on 28 September 2011).

(c) 2011 by the authors; licensee MDPI, Basel, Switzerland. This article is an open access article distributed under the terms and conditions of the Creative Commons Attribution license (http://creativecommons.org/licenses/by/3.0/.) 